\title{
Optimization of Specimen Preparation Methods for Cryo Electron Microscopy of Oil-in-water Emulsions
}

\author{
Dalaver H. Anjum ${ }^{1}$, Sandra Medina ${ }^{2}$, Ali R. Behzad ${ }^{1}$, Seyedeh A. Tabatabai ${ }^{2}$ and TorOve Leiknes ${ }^{2}$ \\ ${ }^{1 .}$ Imaging and Characterization Lab, King Abdullah University of Science \& Technology (KAUST), \\ Saudi Arabia \\ 2. Water Desalination and Reuse Center, King Abdullah University of Science \& Technology (KAUST), \\ Saudi Arabia
}

Emulsions are very important to several key industries such as water treatment (i.e. municipal/industrial wastewater), pharmaceutical, and petroleum [1,2]. Crude oil is typically extracted from oil-wells by injecting water into the reservoir resulting in an extracted flow of water-oil-gas mixture. The water from the extraction process is referred to as produced water (PW). During the separation process both waterin-oil (W/O) and oil-in-water $(\mathrm{O} / \mathrm{W})$ emulsions are formed. The $\mathrm{O} / \mathrm{W}$ emulsions are not readily removed during the oil and gas separation processes and remain in the PW, making discharge or reuse of the PW a challenge. Thus, a more efficient removal of the $\mathrm{O} / \mathrm{W}$ emulsions is necessary to minimize their unwanted effects on the environment. However, the removal of oil from water demands a full understanding of oil and water mixing in these emulsions. In this regard, cryo scanning electron microscopy (cryoSEM) and cryo transmission electron microscopy (cryoTEM) can be utilized to obtain more detailed information about the emulsions with nanometer range resolutions [3]. There is still a need to address the effects of specimen preparation methods on the successful imaging of emulsions with these techniques. This study presents an investigation of sample preparation strategies for improved SEM and TEM analysis to characterize O/W emulsions in PW. Emulsions were synthesized by mixing $\sim 1 \%$ crude oil in water and then stabilizing them with sodium dodecyl benzene sulfonate (SDBS) surfactant. The specimens for cryoSEM were prepared by using plunge and high-pressure freezing (HPF) methods. Whereas the cryoTEM specimens were prepared with an automated plunge freezing system (Vitrobot ${ }^{\mathrm{TM}}$ from FEI Company). SEM and TEM analyses were performed by utilizing Nova nanoSEM and Titan Krios instruments from FEI Company, respectively.

CryoSEM analysis of specimens frozen with both plunge and HPF methods yielded similar results (Figure 1A). From those results, it was observed that most of the oil had left the specimens during the water sublimation process (a pre-requisite for high quality cryoSEM analysis). As a result, only few oil droplets along with some smudges were left behind to image (e.g. the circled area in Figure 1A). To get around this problem, the emulsions were mixed in agarose in the hope that it would help retain their morphology. Plunge frozen specimens of agarose only were first analyzed to get an understanding of the agarose morphology. As seen from the image presented in Figure 1B, the agarose formed a filament-like morphology with irregular-size pores. The plunge freezing of agarose with emulsions was then conducted and a representative image of their specimens is shown in Figure 1C. Basically, a similar smudges-like structures (enclosed by dotted circle in Figure 1C) of oil were observed along with agarose. The structures observed were very similar to the ones presented in Figure 1A, thus the plunge freezing of agarose with emulsions did not allow a successful SEM analysis. Samples were then frozen with the HPF method and a SEM image of one of the specimens is shown in Figure 1D. Results show that the quality of HPF specimens was excellent since the spherical morphology of oil droplets was 
nicely retained. On cryoTEM observations, the results from plunge frozen emulsions (without agarose) are shown in Figure 1E and various size droplets were found to be present therein. Their higher magnification image is presented in Figure 1F, revealing the formation of droplets as small as $10 \mathrm{~nm}$. It is a remarkable result and demonstrates that SDBS surfactant effectively helps in stabilizing the emulsions. The sizes of droplets present in SEM and TEM images were measured to obtain droplets' particle-size distributions (PSDs) and the obtained results are shown in Figure 2. It was found out that the average size of droplets in SEM images was around $120 \mathrm{~nm}$ while it was around $35 \mathrm{~nm}$ in TEM images.

In summary, a two-step recipe for a successful SEM analysis of O/W emulsions was presented in this report. It involves the mixing of emulsions in agarose and then freezing the mixture with HPF method. Whereas for cryoTEM the automated plunge freezing of emulsions alone was found to be sufficient to acquire desired results. In conclusion, the presented results demonstrate a successful application of cryo electron microscopy in revealing the formation of less than $100 \mathrm{~nm}$ size oil droplets in $\mathrm{O} / \mathrm{W}$ emulsions which is otherwise rather difficult, if not impossible altogether, using optical microscopy.

References:

[1] J. C. Schwarz et al, J. Pharm. Sci. 100 (2011), p. 2911.

[2] R. Zolfaghari et al, Separation and Purification Technology 170 (2016), p. 377.

[3] H. S. Lee et al, Langmuir 30 (2014), p. 10826.

Figure 2

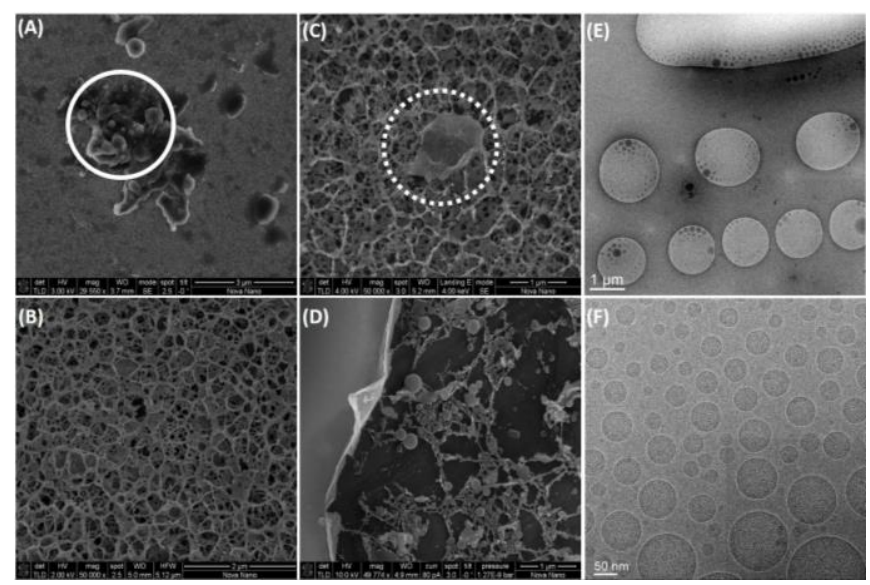

Figure 1

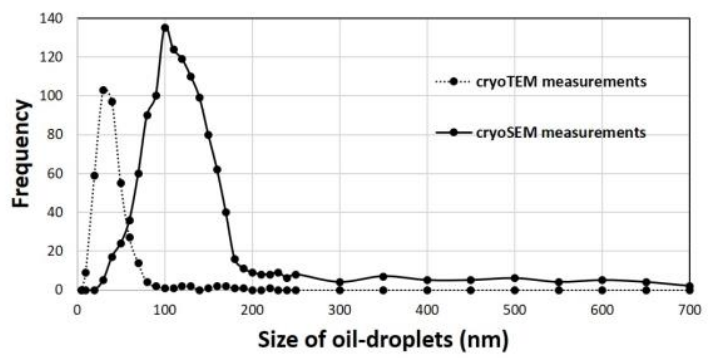

Figure 1. CryoSEM and cryoTEM analyses of crude oil emulsion. (A): SEM image of emulsion frozen with HPF method. (B) SEM image of agarose alone. (C) SEM image of a plunge frozen agarose+emulsion specimen. (D) SEM image of a HPF frozen agarose+emulsion specimen. (E) BFTEM image of emulsion specimen. (F) Higher magnification BF-TEM image of emulsion.

Figure 2. PSDs of oil droplets determined from the SEM and TEM analyses of O/W emulsions. 